\title{
A Variety of Atherosclerotic Lesions in the Aortoiliac Wall Visualized by Non-obstructive Angioscopy
}

\author{
Nobutaka Masunaga, MD, $\mathrm{PhD},{ }^{1 *}$ Yoshitaka Iwanaga, $\mathrm{MD}, \mathrm{PhD},{ }^{2}$ \\ Seijiro Shimada, MD, PhD, ${ }^{1}$ and Junkichi Hama, $\mathrm{MD}, \mathrm{PhD}^{1}$ \\ ${ }^{1}$ Department of Cardiology, Kindai Sakai Hospital, Sakai, Osaka, Japan \\ ${ }^{2}$ Department of Cardiology, Kindai University Hospital, Osakasayama, Osaka, Japan
}

\begin{abstract}
The existence of aortoiliac atheroma is highly associated with aortoiliac aneurysm, dissection, and peripheral arterial embolism. Accordingly, it is crucial to clarify the mechanism and process of the atherosclerotic plaque progression in the aorta and iliac arteries. Here, we report visualization of various plaques along with the entire aorta and iliac artery by non-obstructive angioscopy in a 45 -year-old man with coronary artery disease (CAD). Especially, the significant ulcerative lesions below the infra renal aortoiliac wall were observed, one of which was like a tiny fish mouth opening and closing with the heartbeats. The angioscopic observation on the aortoiliac wall may provide the clue of the atherosclerotic plaque progression.
\end{abstract}

Key words: aortoiliac atheroma, non-obstructive angioscopy, coronary artery disease

\section{Introduction}

Non-obstructive aortic angioscopy is an intravascular imaging modality to acquire real light-based detailed images of the inner surface of the aortic lumen in vivo, which are not well detected by other imaging modalities including computed tomography (CT), magnetic resonance, or ultrasound imaging. ${ }^{1)}$ Non-obstructive aortic angioscopy may clarify the process of atherosclerotic plaque progression in aortoiliac diseases.

\section{Case Report}

A 45-year-old man was admitted to Kindai Sakai Hospital because of stable, exertional chest discomfort. He had no intermittent claudication. His coronary risk factors were hypertension, dyslipidemia, diabetes mellitus, current smoking, and obesity. He had a history of percutaneous coronary intervention (PCI) to right and left circumflex coronary arteries 5 years ago and had been taking aspirin, clopidogrel, bisoprolol, amlodipine, rosuvastatin, ezetimibe, and ethyl icosapentat

\footnotetext{
* Department of Cardiology, Yao Municipal Hospital, 1-3-1 Ryuge-cho, Yao, Osaka 581-0069, Japan

E-mail: nmas1103@yahoo.co.jp

(Received: 2019.12.5; Accepted:2020.4.28)

doi: 10.15791/angioscopy.cr.20.0032
}

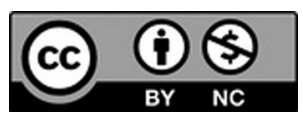

This work is licensed under a Creative Commons [Attribution-NonCommercial 4.0 International] License.

(C) 2020 Japanese Association of Cardioangioscopy since then. There was no calcification in the aorta in chest $\mathrm{X}$-ray and no abnormal Q wave and ST-T change in electrocardiogram. The ankle-brachial index test showed the normal results in both limbs.

The procedure was performed by the right femoral artery approach. Coronary angiography was performed, and severe stenoses were found in mid left anterior descending artery and mid left circumflex artery, where drug-eluting stents were deployed. Subsequently, non-obstructive angioscopy was performed to the whole aorta after observation of the coronary arteries. It was performed by injecting low-molecular-weight dextran from the 4-Fr probing catheter to obtain a clear visual field, ${ }^{1,2)}$ using a 2.25 -Fr angioscopic catheter (INTER-TEC MEDICALS, Japan) while a 6-Fr guiding catheter was slowly pulled back and rotated for vessel-wide screening from the ascending aorta to right external iliac artery.

Irregular surfaces, and white or light-yellow plaques from the ascending aorta to thoracic descending aorta, were observed. In the infra-renal aorta, the dark yellow plaque, small fissure, erosion, various ulcerative lesions with bleeding and scatter like cotton or dandelion fuzz were confirmed (Fig. 1). Subsequently, a significant ulcerative lesion was found in the right common iliac artery just below the bifurcation, which looked like a swelling vulnerable ruptured plaque and formed the fish-mouth-like appearance. It was approximately $2 \mathrm{~mm}$ in diameter and opening and closing along with the heartbeats (Fig. 2 and Video; The video is available online). The enhanced CT showed no remarkable findings (normal diameter and minimum irregular inner 

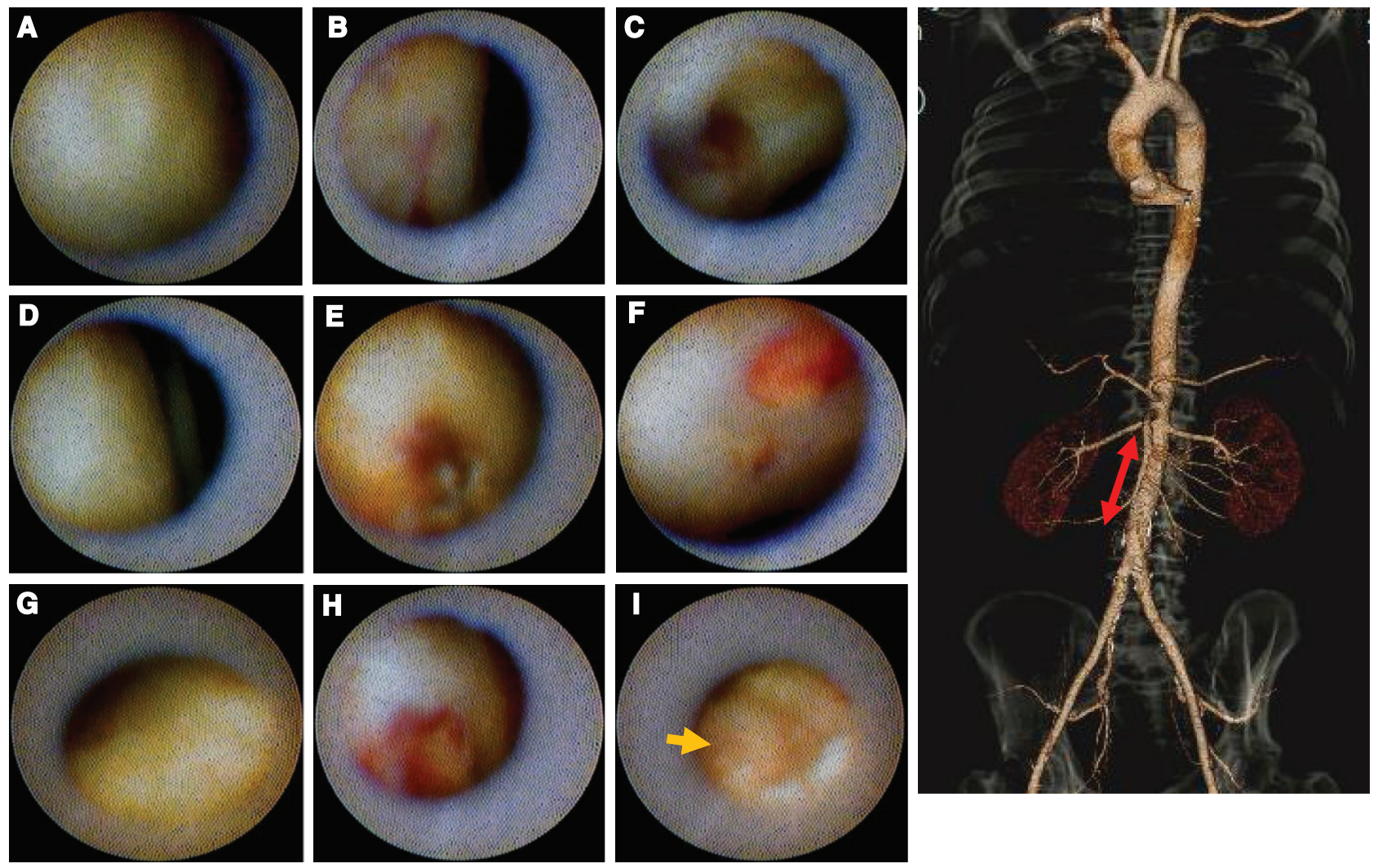

Fig. 1 Non-obstructive angioscopic findings of the infra-renal aorta. Various plaques including light (A) and dark (G) yellow plaques, erosion (B), ulcer-like lesion (C,E,F,H), fissure (D), and scatter like cotton or dandelion fuzz (I, orange arrow) were observed.

surface) in the entire aortoiliac arteries including the portion in the right common iliac artery (Fig. 3).

No clinical events such as formation of aortic aneurysm, aortic dissection, or distal embolism have occurred during 2 years after the examination.

\section{Discussion}

The existence of aortoiliac atheroma is highly associated with aortoiliac aneurysm, dissection, and peripheral arterial embolism. ${ }^{3)}$ Currently, it is not obvious whether and how the atherosclerotic lesions may progress to overt aortoiliac diseases such as aneurysm formation and dissection. Accordingly, it is crucial to clarify the mechanism and process of the atherosclerotic plaque progression in the aorta and iliac arteries. Angioscopy has been reported to be useful for the direct visualization of thrombus and for the characterization of inner surface property of the arterial wall. ${ }^{1)}$ The angioscopic observation on the aortoiliac wall might provide the clue of the atherosclerotic plaque progression, which may not be clarified by the conventional modalities.

The ulcerative lesion in the portion of right common iliac artery might be regarded as a penetrating atherosclerotic ulcer (PAU) in the aortoiliac wall. ${ }^{4)}$ Stanson et al. ${ }^{3)}$ defined PAU as an atherosclerotic lesion with an ulceration that penetrates the internal elastic lamina and the media of the aortic wall histologically. However, we had better regard this tiny fish mouth as an ulcer-like lesion rather than PAU because there was no histopathologic proof and the lesion depth was uncertain. Generally, atheromatous ulcers develop in patients with advanced atherosclerosis. In the initial stages, the lesions are usually asymptomatic and confined to the intimal layer. These lesions can progress to a deep atheromatous ulcer that penetrates through the elastic lamina and into the media. Once formed, the ulcer may remain quiescent or the pathological process may progress, with several potential complications such as dissection, aneurysm formation, or aortic rupture. ${ }^{4}$ This lesion might be an original lesion in such a progressive aortoiliac disease. Indications for intervention for PAU include refractory or recurrent symptoms, hemodynamic abnormality, penetration of the lesion through the aortic wall, aortic diameter expansion, PAU diameter and depth, and the presence of saccular aneurysm. ${ }^{5,6)}$ At this time, we decided that any intervention was not necessary in this case since the lesion was so tiny and no remarkable findings were detected by CT. Recently, Flohr et al. ${ }^{7)}$ reported the natural history of penetrating ulcers of the abdominal aorta and iliac arteries and they performed the conservative management for the asymptomatic patients. We should also perform the medical and life-style management to this patient to prevent the progression as intensively as we can.

The purpose of arterial observations by angioscopy is to evaluate local arteriosclerotic lesions and the therapeutic effect on the artery after intervention. Screening of the 

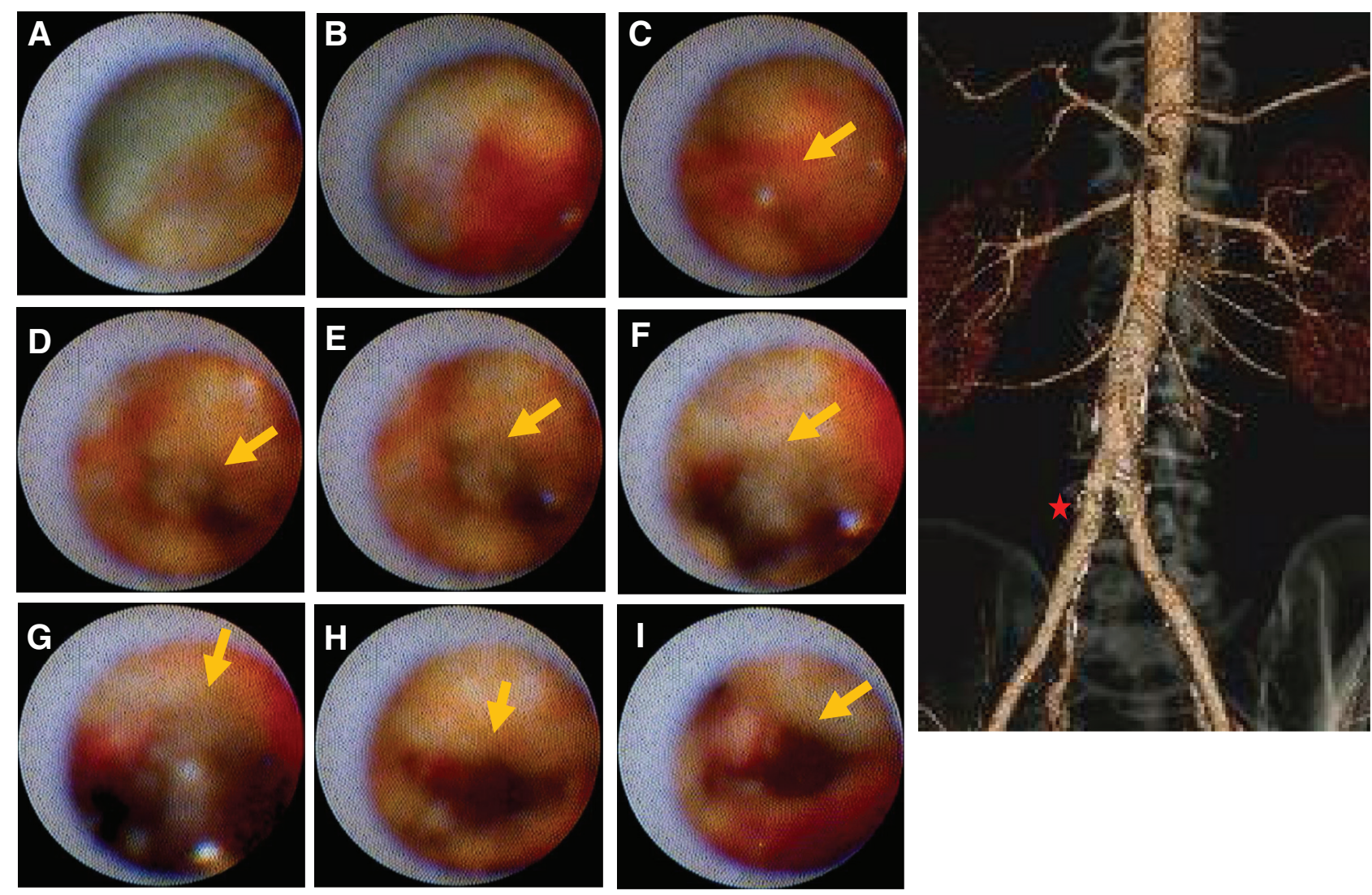

Fig. 2 and Video Ulcerative lesion like a tiny fish mouth in the right common iliac artery. Ulcerative lesion like a tiny fish mouth which opened and closed following the heartbeats was observed in the right common iliac artery (red star). Orange arrows show a mouth of the tiny ulcerative lesion. $\mathrm{C}$ and $\mathrm{G}$ show the closing mouth and opening mouth, respectively (The video is available online).

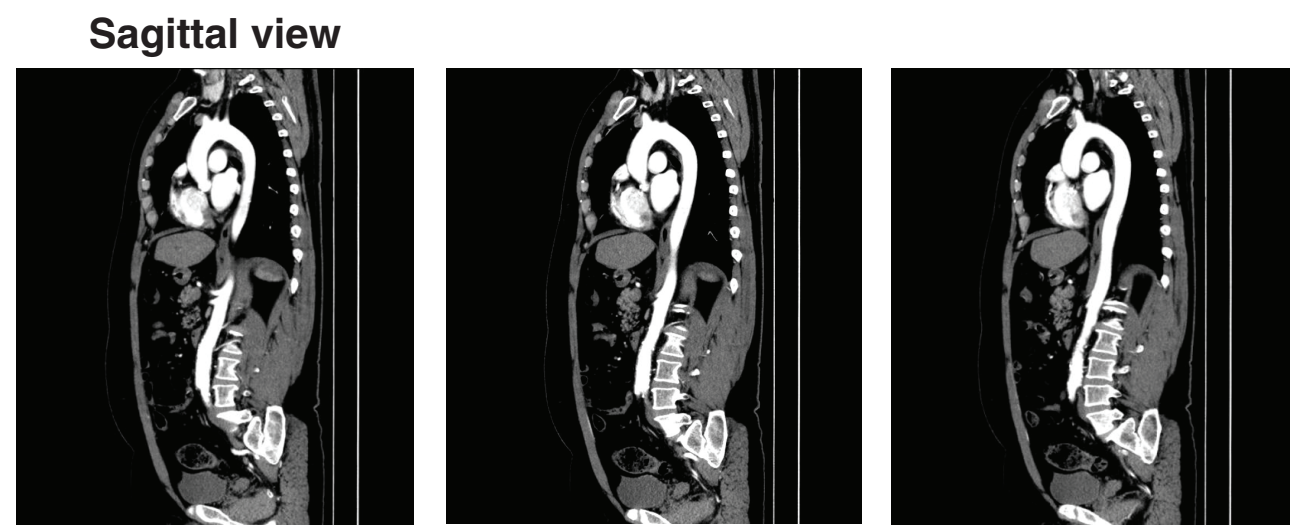

\section{Coronal view Cross sectional view}
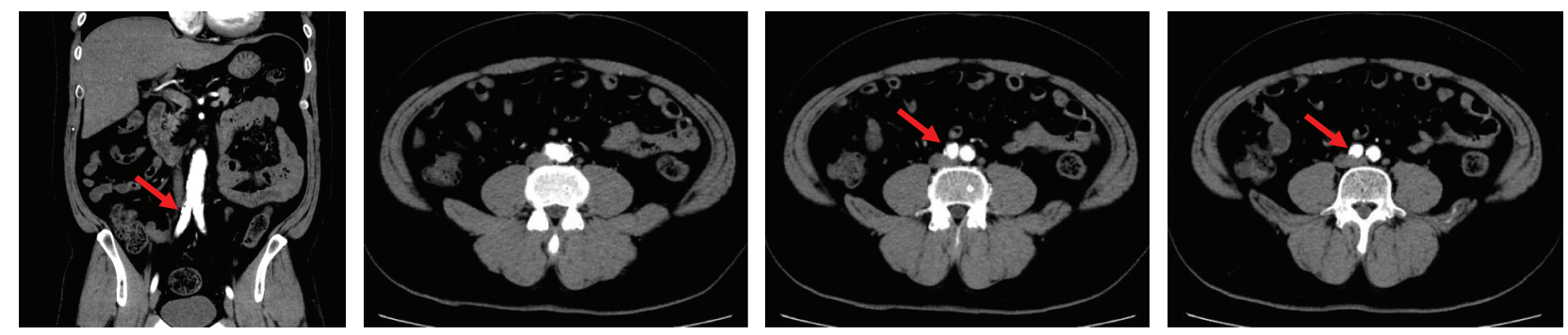

Fig. 3 Enhanced CT findings in the aorta and right common iliac artery. The enhanced CT showed that normal diameter and minimum irregular inner surface in the aorta and in the portion of right common iliac artery (red arrow).

CT: computed tomography 
entire thoracic and abdominal aorta is also indicated as a method for identifying aortic plaques that causes atheromatous embolization and aortic cerebral infarction. Since a close relationship between coronary artery disease (CAD) and aortic atherosclerosis has been reported and the combination shows poor prognosis, ${ }^{89}$ ) we performed the aortic angioscopy in this case with severe CAD. Aortic atherosclerotic plaques can be quantitatively evaluated by nonobstructive angioscopy and its risk evaluation may be considered in the future. Although a femoral artery approach like this case can provide continuous information about entire aorta, the information may be limited in case of the radial artery approach. ${ }^{2)}$ To date, no serious complications causing atheromatous embolization by the procedure itself have been reported, but it should be performed with great care under the guidance of a well experienced facility. ${ }^{2}$

In a 45-year-old man with $\mathrm{CAD}$, we visualized various plaques along with the entire aorta and iliac artery and the significant ulcerative lesions below the infra renal aortoiliac wall by non-obstructive angioscopy. Angioscopy was more useful for detection of atherosclerotic plaques in the aortoiliac wall than other imaging modalities such as CT. We need to accumulate such cases and try the pathophysiological elucidation of aortoiliac atheroma using angioscopy.

\section{Acknowledgments}

We would like to thank Dr. Takahiro Matsumoto and Ms. Yoshie Kamei for their technical assistance.

\section{Disclosure Statement}

There are no conflicts of interest.

\section{References}

1) Komatsu S, Ohara T, Takahashi $S$, et al: Early detection of vulnerable atherosclerotic plaque for risk reduction of acute aortic rupture and thromboemboli and atheroemboli using non-obstructive angioscopy. Circ J 2015; 79: 742-750

2) Hiro T, Komatsu S, Fujii H, et al: Consensus standards for acquisition, measurement, and reporting of non-obstructive aortic angioscopy studies: a report from the working group of Japan vascular imaging research organization for standardization of non-obstructive aortic angioscopy (Version 2017). Angioscopy 2018; 4:1-11

3) Stanson AW, Kazmier FJ, Hollier LH, et al: Penetrating atherosclerotic ulcers of the thoracic aorta: natural history and clinicopathologic correlations. Ann Vasc Surg 1986; 1: 15-23

4) Singhal P, Lin Z: Penetrating atheromatous ulcer of ascending aorta: a case report and review of literature. Heart Lung Circ 2008; 17: 380-382

5) Sato M, Imai A, Sakamoto H, et al: Abdominal aortic disease caused by penetrating atherosclerotic ulcers. Ann Vasc Dis 2012; 5: 8-14

6) Brinster DR: Endovascular repair of the descending thoracic aorta for penetrating atherosclerotic ulcer disease. J Card Surg 2009; 24: 203-208

7) Flohr TR, Hagspiel KD, Jain A, et al: The natural history of penetrating ulcers of the iliac arteries. J Vasc Surg 2016; 63: 399-406

8) Rohani M, Jogestrand T, Ekberg M, et al: Interrelation between the extent of atherosclerosis in the thoracic aorta, carotid intima-media thickness and the extent of coronary artery disease. Atherosclerosis 2005; 179: 311-316

9) Aono J, Ikeda S, Katsumata Y, et al: Correlation between plaque vulnerability of aorta and coronary artery: an evaluation of plaque activity by direct visualization with angioscopy. Int J Cardiovasc Imaging 2015; 31: 1107-1114 